Conclusion In AMI patients treated with primary PCI, Combination of thrombus aspiration and tirofiban was safe and effective, which could lower thrombosis burden, improve distal myocardium perfusion and cardiac function after procedure, did not increase the incidence of MACEs.

\section{e0483 THE CLINICAL RESEARCH FOR ENDOVASCULAR TREATMENT OF DEBAKEY III AORTIC DISSECTION RECEIVED DOMESTIC THIN STEEL BINDING STENTS GRAFTING}

doi:10.1136/hrt.2010.208967.483

Liao Wei, Zhou Aiqin. The Affiliated Hospital of Gannan Medical College

Objective To evaluate the efficacy and safety of endovascular treatment for Debakey III aortic dissection by domestic thin steel Binding stents grafting.

Methods Between October 2006 to March 2010, 42 patients (all male, average age $(53.5 \pm 12.8)$ years, range $43 \sim 70$ years old) with Debakey III aortic dissection was treated with domestic thin steel Binding stents grafting, used an innovative transmission and delivery methods in all patients, of which branch stent were inplanted when the distance from the break of descending aorta to left subclavian artery is less than $10 \mathrm{~mm}$.

Results 42 patients were successfully implanted 48 thin steel Binding stents, including four branch stents. After the operation, six patients were verified endoleak, 3 of the patients were resolved by repeated stent distension and 3 cases were treated by placement of another stent. Three months later, one patient showed new rupture at the remote port of the stent and then was successfully implanted a new stent. Four cases showed numbness of right lower extremity and 1 case showed intermittent claudication of it. In follow-up of 3-32 months, MRI or CT showed the reduce of the false cavity with the formation of intraluminal thrombus, the enlargement of true cavity, and no complications such as tumour rupture, internal leakage and stent displacement in all of the patients.

Conclusions Endovascular thoracic aorta repair, with domestic thin steel Binding stents grafting and the innovative methods of interventional therapy, is an effective, less invasive and safe surgery with faster postoperative recovery, higher success rate and fewer complications for patients with Debakey III type aortic dissection, especially applicable to high-risk patients.

\section{e0484 THE SAFETY STUDY OF TIROFIBAN IN PATIENTS WITH STEMI DURING EMERGENCY PCI}

doi:10.1136/hrt.2010.208967.484

Guo Lai-jing, Hu Dayi, Huo Yong, Tang Oiang, Zhang Huiying, Xing Liying, Wang Zhi, Zhang Shuhe, Shi Zhentao, Zhu Zhenzhong. Chronic Disease Institute, Beijing University Shougang Hospital

Objective To assess the safety of GP IIb/IIIa antagonists tirofiban in patients with ST elevation myocardial infarction (STEMI) during emergency Percutaneous Coronary Intervention (PCI).

Method 120 patients with STEMI during emergency PCI, divided into two groups, tirofiban+PCI group $(n=60)$ and primary PCI group $(n=60)$. The two groups are compared on their major adverse cardiovascular events (MACE) rates which consisted of death, new onset myocardial infarction, persistent myocardial ischeamic state, Cardiac function (1eft ventricular ejection fraction) after operation, compression haemostasia time and adverse drug effect while in hospital.

Result Major adverse cardiovascular events (MACE) rates in tirofiban + PCI group was higher than that of primary PCI group $(10 \%$ vs $6.7 \%$ ); Postoperative heart function was better in tirofiban $+\mathrm{PCI}$ group than that in primary PCI group (56.97 \pm 8.41 vs $54.15 \pm 7.11$ ): There was no difference in severe haemorrhage between the two groups. The bleeding event rates were of higher tendency in tirofiban+PCI group. ( $8.3 \%$ vs $3.3 \%$ ). APTT and compression haemostasia time of tirofiban + PCI group were significant when prolonged $(53.97 \pm 10.58$ vs $32.51 \pm 6.31) \mathrm{p}<0.05)$, but no more bleeding and heamatom events occurred during prolongation.

Conclusion GPII b/III a antagonists tirofiban+PCI is a possible safe and effective reperfusion method with STEMI during emergency PCI.

\section{e0485 CLINIC STUDY OF DOMESTIC TIROFIBAN ON TIMI FLOW DURING FACILITATED PCI}

doi:10.1136/hrt.2010.208967.485

${ }^{1}$ Guo Lai-jing, ${ }^{1} \mathrm{Hu}$ Dayi, ${ }^{1}$ Huo Yong, ${ }^{1}$ Tang Qiang, ${ }^{1}$ Zhang Huiying, ${ }^{1}$ Xing Liying, ${ }^{1}$ Wang Zhi, ${ }^{2}$ Ou Huaqing, ${ }^{1}$ Shi Zhentao, ${ }^{3}$ Zhu Zhenzhong, ${ }^{1}$ Yan Do, ${ }^{1}$ Tang Ounzhong, ${ }^{1}$ Chen Xuezhi, 'Liu Xiumin. ${ }^{1}$ Department of Cardiology Beking University Shougang Hospital; ${ }^{2}$ Department of Cardiology Beking University Shougang Hospital; ${ }^{3}$ Department of Emerergency Beking University Shougang Hospital

Objective To assess Thrombolysis In Myocardial Infarction (TIMI) flow of the nation-produced tirofiban in patients with ST-segment elevation myocardial infarction (STEMI) during primary Percutaneous Coronary Intervention (PCI).

Methods 120 patients with STEMI during primary PCI from Nov 2006 to June 2009 were divided into two groups, tirofiban $+\mathrm{PCI}$ group of 60 cases (51 males and 9 females) with mean age $(60.11 \pm 10.96)$ years old, and the other primary PCI group of 60 cases (42 males and 18 females) with mean age $(64.33 \pm 11.91)$ years. The TIMI flow before and after operation were observed in all cases angiographically.

Results By using tirofiban before operation improved TIMI flow, a greater percentage of TIMI 1 grade flow was achieved in the tirofiban $+\mathrm{PCI}$ group compared with the primary PCI group before operation (13.3.8\% vs 3.3\%, p>0.05). There was no difference of TIMI 3 grade flow between the two groups after operation, TIMI 2 grade flow was lesser in tirofiban $+\mathrm{PCI}$ group $(6.7 \%$ vs $3.4 \%$, $\mathrm{p}>0.05)$. Reperfusion arrhythmia was lesser in tirofiban $+\mathrm{PCI}$ group (3.4\% vs $6.7 \%)$.

Conclusion Tirofiban may improve TIMI flow of the IRA in patients with STEMI during emergency PCI.

\section{e0486 TRANSCATHETER CLOSURE OF NO RIM LARGE ATRIAL SEPTAL DEFECT WITH AMPLATZER OCCLUDERS TECHNICAL CONSIDERATIONS, SAFETY AND FEASIBILITY}

doi:10.1136/hrt.2010.208967.486

Chen Lianglong, Luo Yukun, Lin Chaogui, Peng Yafei, Zheng Xingchen. Fujian Union Hospital

Backgrounds Transcatheter closuring no rim or large atrial septal defect (ASD) with Amplatzer occluding device (AOD) was technically challenged. The present study was to address technical issues, and to test the safety and feasibility for transcatheter closuring large and no rim ASDs with AOD.

Methods Patients, with large ASDs of $325 \mathrm{~mm}$ and with no rims at least in one defect border detected echocardiographically, were included in the study. 49 patients eligible underwent transcatheter closure of ASDs and divided into group A ( $n=26$, large ASDs with intact rims) and group $B(n=23$, large ASDs with no rims). Three occluding methods i.e. the conventional releasing, the waist prereleasing, and the dumbbell-shaped releasing were sequentially 